\title{
Cosmic ray triggering using muon signal detected by the Hadronic Calorimeter of ATLAS
}

\section{B. C. Ferreira ${ }^{* \dagger}$}

Federal University of Rio de Janeiro/COPPE

E-mail: bcarneirodlps.ufrj.br

\section{J. M. de Seixas}

Federal University of Rio de Janeiro/COPPE

E-mail: seixas@lps.ufrj.br

\section{A. S. Cerqueira}

Federal University of Juiz de Fora

E-mail: santiago@lacee.ufjf.br

This paper presents the development of a matched filter system for muon detection using the muon output trigger signal, provided by the hadronic calorimeter of ATLAS detector, the Tile Calorimeter.

A signal processing is done for this muon output to improve its signal-to-noise ratio and detection capability. The performance achieved for this system, using data from the testbeam period, was $96 \%$ of detection efficiency against a false alarm of $10 \%$. This performance encourages the use of the muon output together with the matched filter system for triggering cosmic rays, during the commissioning phase the ATLAS detector.

XI International Workshop on Advanced Computing and Analysis Techniques in Physics Research April 23-27 2007

Amsterdam, the Netherlands

\footnotetext{
* Speaker.

$\dagger$ The authors would like to thanks CAPES, CNPq and FAPERJ (Brazil) and CERN (Switzerland) for the support to this work. We would also like to thank our colleagues from the TileCal collaboration for providing the data set and for fruitful discussions concerning this work.
} 


\section{Introduction}

The Large Hadron Collider (LHC) will collide protons beams with a center-of-mass energy of $14 \mathrm{TeV}$ (one order of magnitude higher than the existing accelerators). Furthermore, it will achieve a bunch crossing rate of $40 \mathrm{MHz}$ and a luminosity of $10^{34} \mathrm{~cm}^{-2} \cdot \mathrm{s}^{-1}$ [1], envisaging an extense physics programme (e.g. Higgs particle, SUSY, high precision measurements of Standard Model parameters) [2]. LHC is being installed in the $27 \mathrm{~km}$ tunnel, used by the previous accelerator LEP, buried around 50 to $175 \mathrm{~m}$ underneath the border between France and Switzerland, near Geneva city.

In order to characterize the complex interactions produced by the high-energy beam collision, LHC will have four detectors installed in different collision points. ATLAS [2] is one of the main detectors being developed for LHC, and it has been design for excellent: tracking, electromagnetic and hadronic calorimetry, hermeticity and muon measurements [2].

Due to the high event rate of LHC, approximately $10^{9}$ high multiplicity events/s (at nominal luminosity will be 20-25 events per bunch crossing, i.e. every $25 \mathrm{~ns}$ ), it is essential the use of a sophisticated online triggering system. For the detector ATLAS, the trigger system is divided in three levels of increasing complexity and processing time [3, 4].

The first-level trigger (LVL1) is implemented in customized hardware and has been developed to reduce the initial bunch crossing rate to a maximum of $75 \mathrm{kHz}$ [3]. Its event selection is based on calorimetry (with reduced granularity) and dedicated muon detectors. The next levels of triggering system should reduce the acceptance rate to $100 \mathrm{~Hz}$ [4].

The hadronic calorimeter (TileCal) from ATLAS was design to provide two trigger signals for LVL1 operation: the trigger towers (total energy in pseudorapidity $\mathrm{X}$ azimuthal angle $=0.1 \mathrm{X} 0.1$ ) and the muon output signals (foreseen as an upgrade at the triggering system) [5].

The muon output signal has a very low signal-to-noise ratio, since the muons deposit very small levels of energy on the calorimeter. There are always two muon outputs that are very correlated.

Muons produced in cosmic ray showers are often used to test, align and calibrate detectors in high energy physics. During the ATLAS commissiong phase, that is ongoing, TileCal is providing the trigger for cosmic rays for all the sub-detectors of ATLAS. A part of the trigger towers signals are sent to a coincidence board [6]. This board is responsible to verify if any of the tower signals exceed a certain threshold. So, if any tower from the top part and any tower from the bottom part at the same time exceed the threshold, a trigger is sent for all the detector, and the event is recorded.

In this paper, muon output is pre-processed in order to improve its signal-to-noise ratio and, then, it is sent to the matched filter, that is the optimum filter for detecting a deterministic filter that is corrupted by a additive white Gaussian noise.

\section{The hadronic Tile Calorimeter}

The Tile Calorimeter (TileCal) is a hadronic sampling calorimeter which makes use of iron as the absorber material and scintillating plates, readout by wavelength shifting fibers (WLS), as active medium [5]. The TileCal consists of a cylindrical structure divided in 64 azimuthal segments 


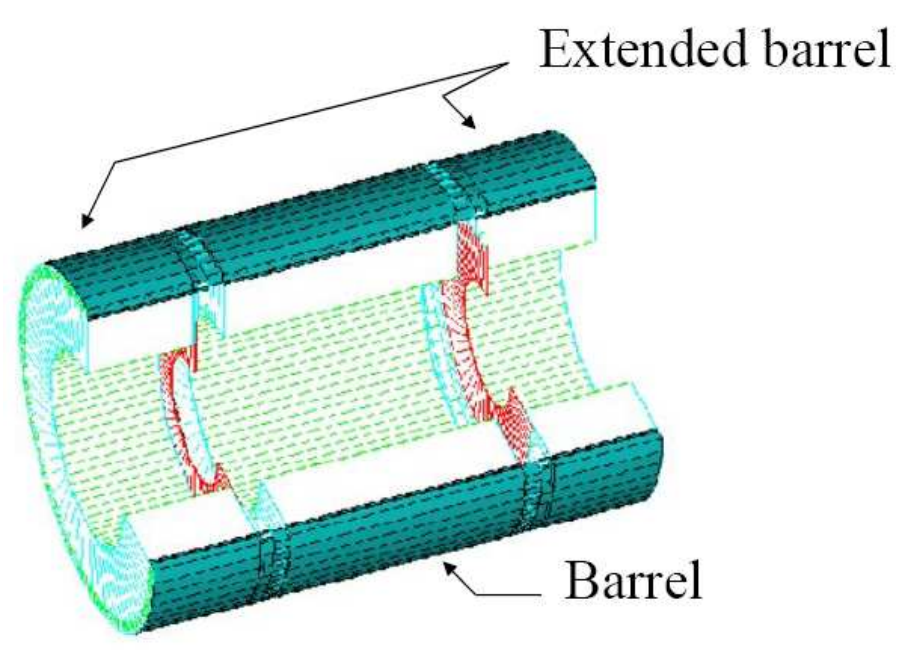

Figure 1: Tile Calorimeter.

(modules). As shown on Figure 1, in the longitudinal direction, TileCal is divided into three parts: the barrel and two extended barrels.

As from Figure 2, each barrel module is segmented into 45 cells (16 cells for the extended barrel modules) with double readout, which means that each cell provides two signals (called left and right readout). On the radial direction, the modules are segmented in three sampling layers (A, B-C and D for the barrel and A, B and D for the extended barrel).

Light signals proportional to the energy of the incoming particle are produced by the scintillating tiles. These signals are transported through the WLS fibers to photomultiplier tubes (PMT), that convert the light signals into and electric signals (see Figure 3). Restricting to the trigger information, the electric signal is then conditioned through a shaper circuit and an analog adder builds both two trigger signals:

- trigger tower ${ }^{1}$ signal, built by combining linearly 5 signals (typically) from the three sam-

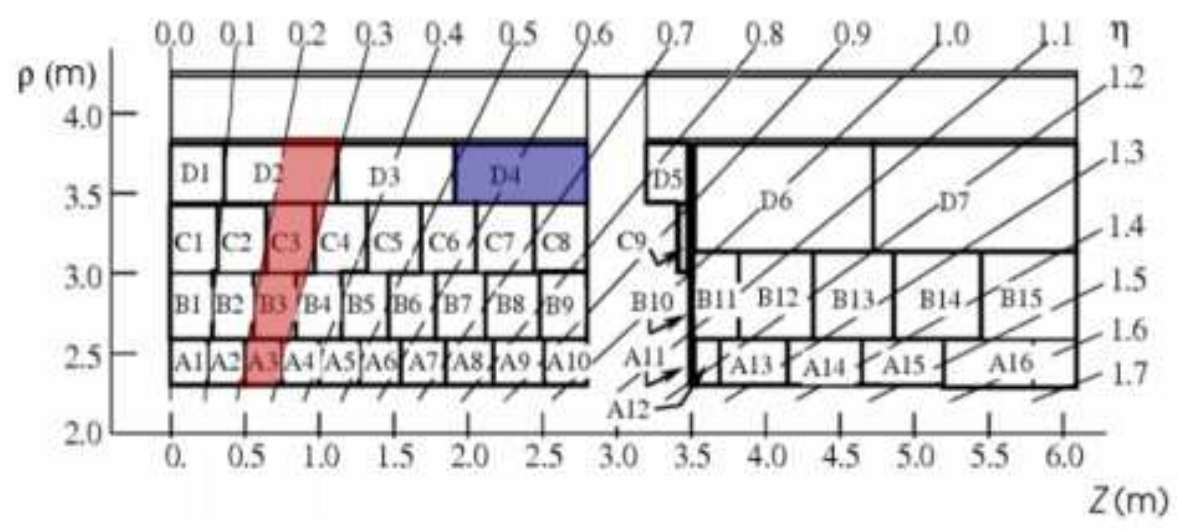

Figure 2: Tile Calorimeter segmentation.

\footnotetext{
${ }^{1}$ region of pseudorapidity $(\eta) \mathrm{X}$ azimuthal angle $(\phi)=0.1 \times 0.1$
} 


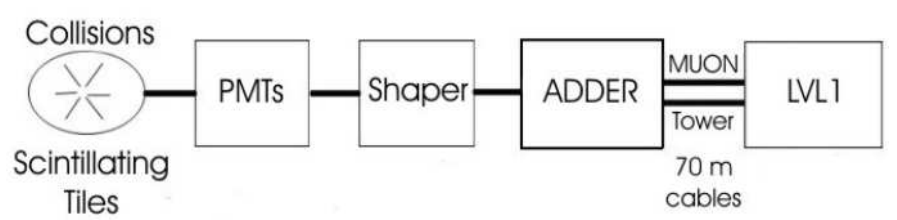

Figure 3: Readout from Tile Calorimeter.

pling layers: 2 from the first, 2 from second and 1 from the last layer (red region of Figure 2);

- muon output signal, which is the amplified replica of the last longitudinal sampling layer signal (blue region of Figure 2).

During the early stages of commissioning, TileCal has been using the tower output as a trigger for cosmics. But, this signals won't be available on the next commissioning phases, as this signals will be going to the LVL1 receptors. An alternative to continuing providing the trigger for cosmics is the use of the muon output trigger signal.

\section{The data set}

The data set used in this work comprises signal and noise runs:

- $180 \mathrm{GeV}$ muon beam, incident at the center of each tower;

- pedestals (data taking without any incident beam).

Both trigger signals, tower and muon output were digitized using an ADC having $40 \mathrm{MHz}$ as the sampling rate and 8 bits of resolution. A total of 16 samples (or $400 \mathrm{~ns}$ ) were acquired per event.

These data sets, were equally split into two: development, which was used for the detector system design, and test set, which was used to evaluate the generalization capability of the design. A total of about 22,000 events was available.

\section{The peak detector}

The peak detector consists in a simple detection technique, which uses only the highest amplitude sample of the incoming signal. The upper left part of Figure 4 shows peak detector histograms for left PMT (one reading from a D-cell). At the bottom part, it is shown the distribution when two PMTs are added up, pointing out a significant improvement in detection efficiency. The detection efficiency improvement can be seen in terms of the Receiver Operating Characteristics (ROC) [9], which is displayed at the right. The ROC curve represents the tradeoff between detection and false alarm, when the decision threshold is varied. For example, for a false alarm of $10 \%$, the detection efficiency increases from around $82 \%$ (using only one readout cell) to more than $90 \%$ (using the two readouts). 


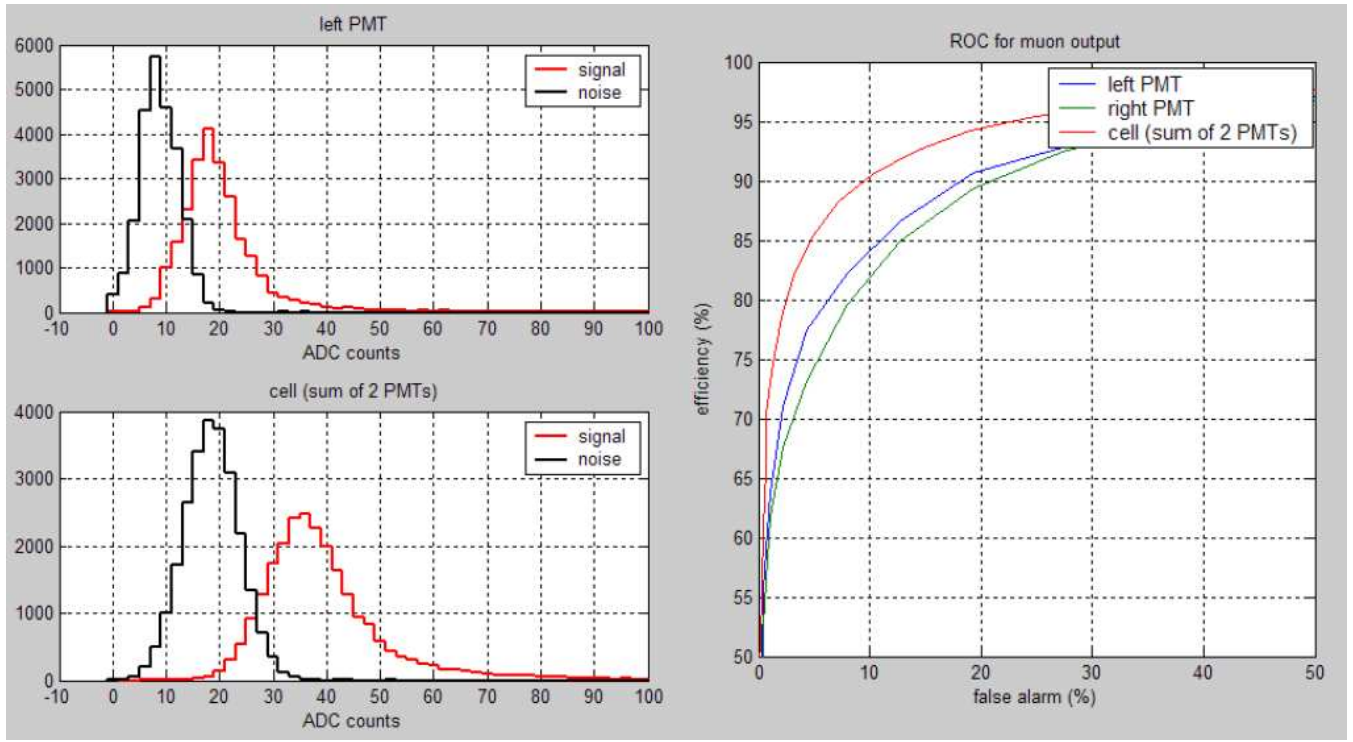

Figure 4: Peak detector efficiency.

\section{The matched filter}

The muon detection problem can be modeled as a classical binary hypothesis making problem [8]

$$
\begin{array}{ll}
H_{1}: & r[k]=s[k]+n[k], k=1, \cdots, N \\
H_{0}: & r[k]=n[k]
\end{array}
$$

where $n[k]$ is considered to be a zero-mean additive white Gaussian noise with variance $N_{0} / 2$ and $s[k]$ is the signal to be detected. The detection is based on analyzing event acquired with $N$ samples.

For this class of problems, the matched filter is known to be the optimum linear filter (in the sense of the signal-to-noise ratio) for detecting a deterministic signal when the additive noise is white [8].

The matched filter output $y[n]$ is given by

$$
y[n]=\sum_{k=-\infty}^{\infty} h[n-k] x[k]
$$

where $h[n]$ is the discrete impulse response of the filter and $x[k]$ is a replica (reflected and shifted) of the signal to be detected, i.e. the muon signal.

\subsection{Whitening filter}

As said before, the matched filter is the optimum filter in terms of SNR only when the desired signal is corrupted by an additive white noise, i.e., the covariance matrix must be diagonal with identic diagonal elements. For our application, the covariance matrix of the background noise, for the development set, can be seen in Figure 5. It's possible to see that the noise process is not white, 


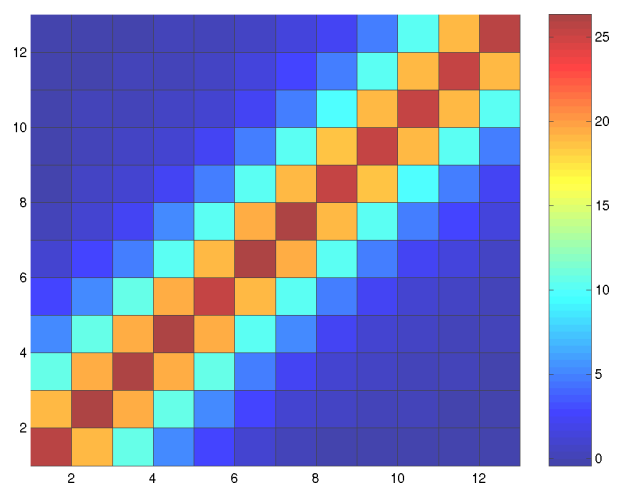

Figure 5: Covariance matrix of the development set.
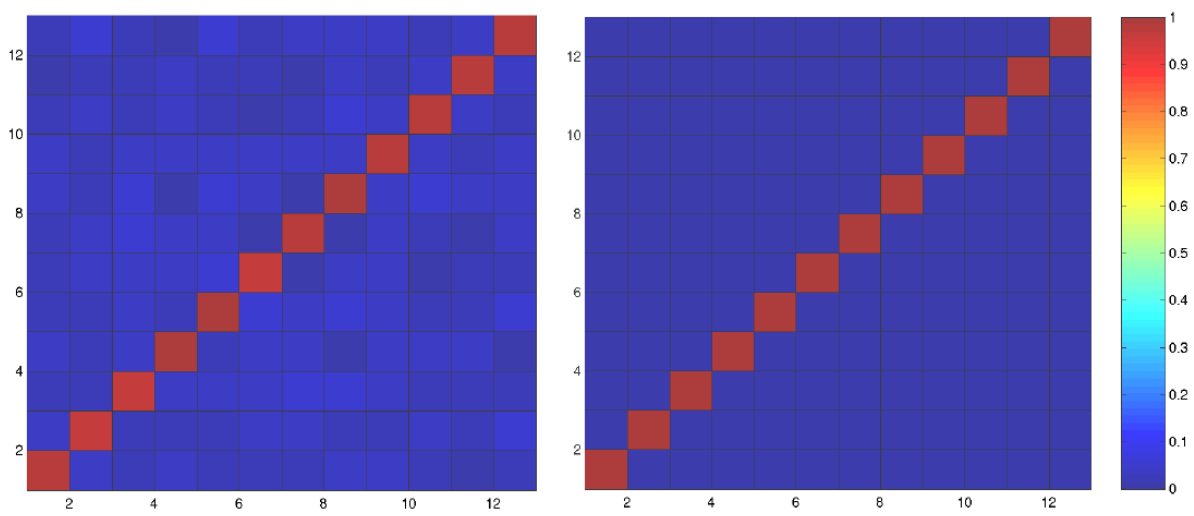

Figure 6: Covariance matrices of the test and development set.

so it's necessary to develop a whitening filter to obtain the optimum performance. The resulting covariance matrices for both development and test sets can be seen in Figure 6, from which the whitening procedure can be considered to generalize well the noise statistics captured from the development set.

\subsection{Results}

The first level trigger of ATLAS has to cope with a high bunch crossing event rate. In order to process the muon output signal as fast as possible, the muon signal is considered a deterministic process. This means that the muon output signal is approximated by the mean value.

The performance of this is shown in Figure 7 in terms of ROC curves. As it can be seen, even using this approximation, the matched filter performance is better than the peak detector. For a false alarm of $10 \%$, the detection efficiency increases from $90 \%$, using the peak detector, to more than $96 \%$, using the whitening filter followed by the matched filter.

\section{Conclusions}

A matched filter system was designed in order to detect muon with the hadronic Tile Calorimeter of ATLAS. This approach uses a pre-processing, by using the sum of the two readouts from the 


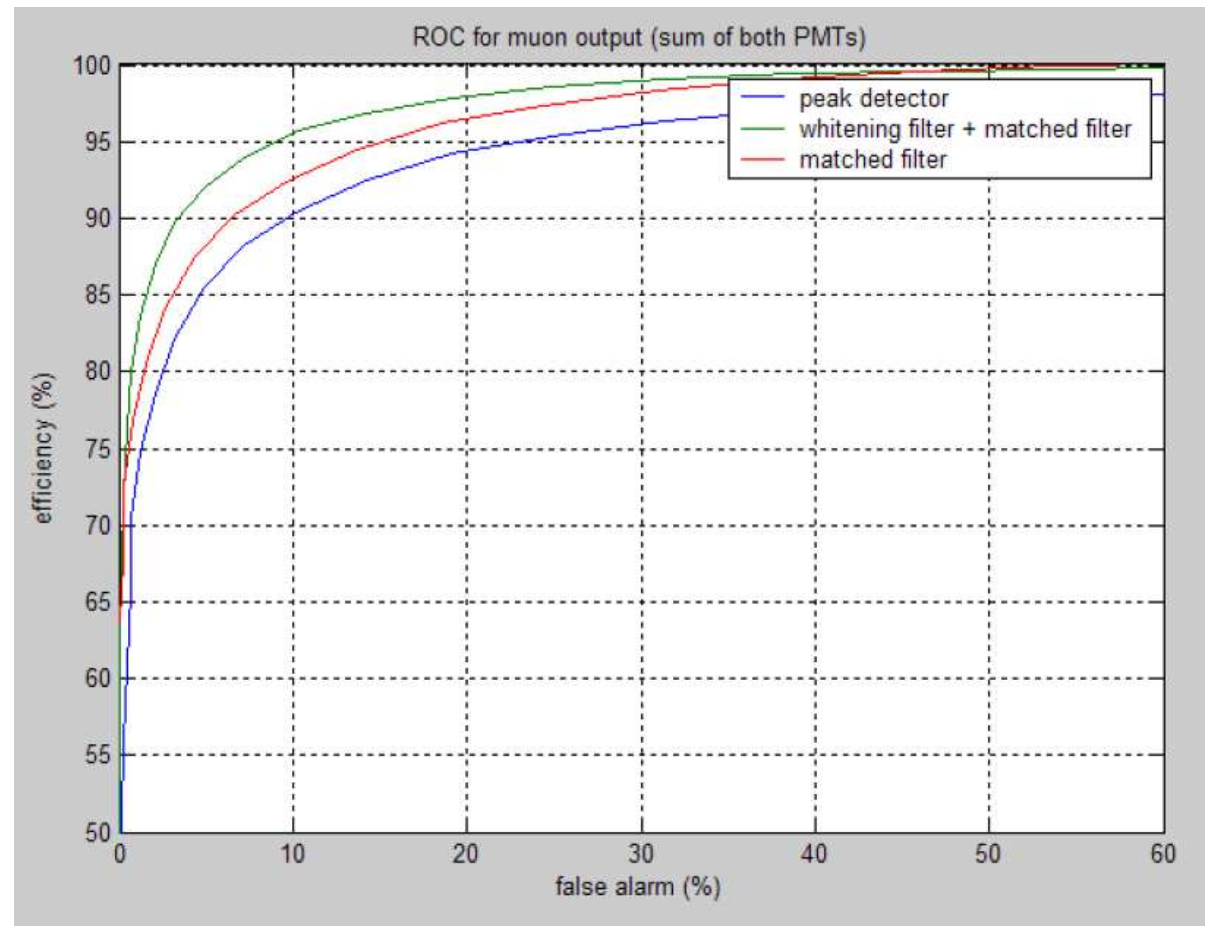

Figure 7: Comparison between peak detector and matched filter (with and without whitening filter).

same D-cell, improving the detection capability. The performance of this system is, for a false alarm of $10 \%$, the matched filter system achieved $96 \%$ of efficiency (when using the whitening filter). The peak detector, for the same false alarm, achieved $90 \%$ of efficiency.

An analog circuit, for summing two readout from the same D-cell is under development and it will be tested soon for cosmic ray triggering during the ATLAS commissioning phase.

\section{References}

[1] LHC - The Large Hadron Collider. Webpage: http://lhc-new-homepage.web.cern.ch/lhc-new-homepage

[2] ATLAS Collaboration: "ATLAS Technical Proposal for a General-Purpose pp Experiment at the Large Hadron Collider at CERN”, CERN/LHCC/94-43, LHCC/P2, 15 December 1994.

[3] ATLAS Collaboration: "Level-1 Trigger". Technical Design Report, ATLAS TDR-12, 1998.

[4] ATLAS Collaboration: "ATLAS High-Level Triggers, DAQ and DCS”. Technical Proposal, CERN/LHCC/2000-17, 2000.

[5] ATLAS Colaboration: "ATLAS Tile Calorimeter Technical Design Report", CERN/LHCC/96-42, ATLAS TDR 3, 1998.

[6] K. J. Anderson et al., "Stand-alone Cosmic Ray Trigger Electronics for the ATLAS Tile Calorimeter", 10th Workshop on Electronics for LHC and Future Experiments LECC 2004, Boston, MA, USA, 2004.

[7] A. S. Cerqueira, T. Davidek and G. Usai, "Tile Calorimeter Muon Trigger Signal", ATL-TILECAL-2002-002, 2002. 
[8] K. S. Shanmugan and A. M. Breipohl, "Random Signals: Detection, Estimation and Data Analysis". John Wiley \& Sons, 1988.

[9] T. Fawcett, "An introduction to ROC analysis", Pattern Recognition Letters, Volume 27, Issue 8 (June 2006). Elsevier Science Inc. 